\title{
A model for PSR J0437-4715
}

\author{
Janusz Gil and Agnieszka Krawczyk \\ Astronomy Centre, Pedagogical University, Zielona Góra, Poland
}

We present the modelling results of full polarization data of the PSR J0437-4715, recently published by Manchester \& Johnston (1995). This pulsar presents a real challenge for pulsar modelling because of seven clear components in its mean profile. In terms of the conal model of pulsar emission this is the first case indicating three distinct cones surrounding the central core beam. We have attempted to model this pulsar and obtained a very satisfactory fit to the data. We succeded to reproduce the complexities of the multiple component profile and polarization properties.

To model the radiation from PSR J0437-4715 we use the method presented in Gil et al. (1995 and references therein). We assume that subpulses in single pulses correspond to the subpulse-associated plasma columns distributed within the overall pulsar beam. Each column represents a gaussian intensity distribution. These columns rotate more or less regularly around the magnetic axis. The exception is the central column directed almost along the magnetic axis defined by the local surface magnetic pole. This column corresponds to the core beam and consequently to the core component in our model. Outer columns rotating at some distances from the core axis will form a number of coaxial conal beams corresponding to the conal profile components. Figure 1a represents a projection of the subpulse-associated columns onto the polar cap while Fig. 1b represents schematically a corresponding multiconal structure of the average beam. The initial positions, the characteristic dimensions and the relative amplitudes of the subpulse spots (Fig. 1a) have been estimated from the mean profile (Fig. 2b). We assume that the subpulse spots are separated from each other by distances comparable with their sizes (Fig. 1a). The very large polar cap with the radius $r_{p}=1.4 \cdot 10^{4} P^{-1 / 2} \mathrm{~cm} \approx 1900 \mathrm{~m}$ as compared with the characteristic subpulse spot dimension $D \approx 300 \mathrm{~m}$ allows three conal rings surrounding the central core beam (Fig. 1b).

Acknowledgments. We thank Dr. R.N. Manchester and Dr. S. Johnston for providing us with the raw data of PSR J0437-4715.

\section{References}

Blaskiewicz M., Cordes J.M., Wasserman J., 1991, ApJ, 370, 643

Gil J., Kijak J., Maron O., Sendyk M., 1995, A\&A, 301, 177

Manchester R.N. \& Johnston S., 1995, ApJ, 441, L65 

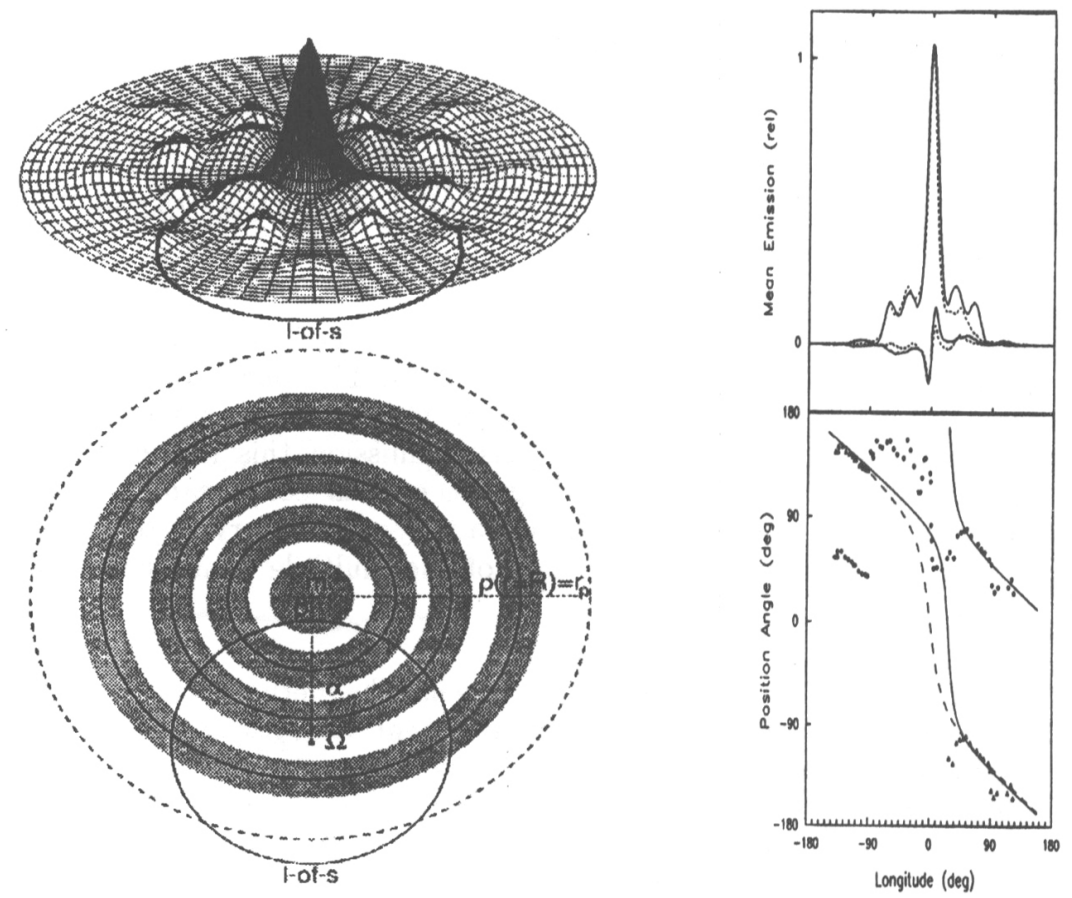

Figure 1. (a) Instantenous arrangement of the polar cap populated by a number of hot spots corresponding to the subpulse-associated plasma columns in the pulsar magnetosphere. (b) Multiple cone structure of the average pulsar beam following from the above. The circle centered on the pulsar spin axis $\Omega$ represents the line-of-sight, which remains within the pulsar beam for about $300^{\circ}$ of longitude. The geometrical parameters which lead to the presented fits are: the inclination angle $\alpha=20^{\circ}$, the impact angle $\beta=-4^{\circ}$ and the opening angle of the beam $\rho=34^{\circ}$. $\quad$ (c) Mean intensity (relative units) and circular polarization for PSR J0437-4715 at $1.4 \mathrm{GHz}$. Solid lines represent the model while dashed lines represent the data. Position angle variations in PSR J0437-4715 observed at $1.4 \mathrm{GHz}$. The open circles represent the raw data while the triangles correspond to $90^{\circ}$ (orthogonal mode transition at the leading edge) or $180^{\circ}$ (trailing edge) rotation. The dashed line corresponds to the classical rotating vector model while solid lines represent relativistic rotating vector model. In the trailing half of the profile the position angle curves (both model and data) are presented twice for clarity. Both solid lines represent the same relativistic (Blaskiewicz et al. 1991) rotating vector model $\left(\alpha=20^{\circ}, \beta=-4^{\circ}\right.$ and $\left.r_{e m}=4.5 \cdot 10^{6} \mathrm{~cm}\right)$. 